\title{
Secuencias y consecuencias de algunos procesos de neolitización en los Andes Centrales
}

Danièle LaVAlléE ${ }^{1}$

\begin{abstract}
RESUMEN
Se describen los principales procesos constitutivos de la "neolitización" andina. El Area Central Andina constituye la primera de Sudamérica donde se ha observado una sedentarización de los grupos humanos previamente a la adopción (o invención) de la agricultura. Desde el 6000 AP existen instalaciones permanentes en el litoral de Perú, orientadas a una explotación óptima de los recursos marinos. En cambio, los más antiguos indicios de especies vegetales manipuladas provienen de cuencas interandinas, en un contexto de caza-recolección y seminomadismo, aunque los centros primarios de domesticación aún no han sido identificados. En cuanto a la domesticación animal, varios focos parecen haber existido en Perú y Chile. Al final aparece la cerámica, último elemento constitutivo del Neolítico andino, probablemente importada desde el norte (Colombia y Ecuador) o desde la foresta tropical oriental.
\end{abstract}

Palabras claves: neolitización-sedentarización-domesticación-agricultura-Perú.

\section{ABSTRACT}

In this paper, are described the main processes of the Andean neolithization. The Central Andean Area represents the first of South America where human groups became sedentary before the adoption (or invention) of agriculture. As soon as the $6000 \mathrm{BP}$, permanent establishments are know on the Peruvian coast, in relation to an optimal explotation of marine ressources. On the other hand, the oldest indices of manipulated vegetal species have been discovered in inter Andean basins, in a context of hunting-gathering and semi-nomadism. The earliest centers of domestication are still to be identified. As for animal domestication, various centers seem to have existed in Peru and Chile. The ultimate element of the Andean Neolithic, ceramics, only appears at the end, probably imported from North (Colombia and Ecuador) or from the Eastern Tropical Forest.

Key words: neolithization - sedentarization-domestication - agriculture-Peru.

Recibido: julio 2004. Aceptado: enero 2006.

1 UMR 8096 “Archéologie des Amériques” (CNRS), Maison de l'Archéologie et de l'Ethnologie, 21 Allée de l'Université 92023 NANTERRE Cedex, FRANCIA. Email: daniele. lavallee@wanadoo.fr
Este trabajo podría también titularse "cambio y continuidad" de acuerdo a que las transformaciones que se producen en el área centro-andina, o mejor dicho que se vuelven visibles en el registro arqueológico, aproximadamente a partir de 8000 AP, se sitúan en un continuит. Empezaré por examinar la naturaleza de estos cambios e intentar definirlos. En el discurso inaugural del Festival Internacional de Geografia realizado en Francia, en el año 2001, la antropóloga Françoise Héritier se interrogaba acerca de las palabras innovación, invención y descubrimiento. Hablando de los procesos prehistóricos andinos, veamos precisamente si se trató de innovaciones, de invenciones o de descubrimientos. Ella sostenía que: "Un descubrimiento señala algo desconocido, pero que ya existe en la naturaleza". Ahora bien, todos los elementos naturales constitutivos de la neolitización andina eran conocidos desde la época arcaica, trátese de la mayoría de las especies vegetales y de las pocas especies animales aptas para la domesticación, o de los materiales y de las técnicas de la construcción arquitectónica monumental, trátese también de la arcilla y sus cualidades plásticas. En este aspecto, los procesos andinos no son consecuencia de uno o varios descubrimientos, pero sí de una nueva manera de utilizar y transformar consecuentemente los elementos ya conocidos. En cambio, "la invención crea algo nuevo gracias a una nueva combinación de condiciones ya conocidas". Se trata, pues, de un paso conceptual que lleva de cierto tipo de conocimientos racionales a otro; hecho que implica toda una red de reflexiones en la cual desempeñan un papel tanto las experiencias anteriores como las influencias exteriores. La fabricación de objetos en barro cocido fue una invención. En cuanto a la innovación, "representa en el orden de la vida social, económica y política lo que es la invención en el orden de la técnica. Es decir, un cambio radical y, teóricamente, irreversible, de todo lo que se consideraba adquirido hasta la fecha" (Héritier 2001: 9). Podemos decir entonces que los procesos andinos de neolitización fueron innovadores, en la medida en que la mirada de los hombres a un cierto número de elementos naturales, sumado, en 
consecuencia, a su capacidad de manipularlos, los cambios se orientaron definitivamente hacia una mayor trasformación.

Surge inmediatamente una pregunta: ¿Porqué aquí y no allá? ¿Porqué en América solamente los Andes y Mesoamérica constituyeron "áreas nucleares", según la definición que dio Braidwood en 1960? En ambos casos, se trata de amplias regiones en las cuales el efecto combinado de condiciones climáticas y potencialidades económicas favorables ha permitido, más temprano que en el resto del continente, la instauración de un modo de vida basado sobre una economía de producción de los recursos alimenticios, proceso mayor de la neolitización. Pero probablemente también es porque, como lo escribía Franz Boas en 1940 (citado por Héritier 2001: 9):

\section{“... la historia de la humanidad demuestra que los} progresos de la cultura dependen de las oportunidades ofertadas a un grupo dado, de sacar una enseñanza de la experiencia de sus vecinos. Los descubrimientos de un grupo se extienden a otros grupos y cuanto más diversos son los contactos, cuanto más numerosas las oportunidades de aprender".

La "historia" del desarrollo cultural andino cuenta con varias ilustraciones de esta observación. Condiciones naturales favorables, por cierto, pero también imitaciones e impulsos exógenos.

Recordaremos cuáles fueron las principales innovaciones que caracterizan la neolitización: la domesticación vegetal y animal, la sedentarización y jerarquización de las sociedades, con todos sus corolarios en el campo de la cultura material, desde la arquitectura pública a los contextos funerarios, y se les adjunta generalmente el uso de la cerámica. Pero estos son componentes comunes a todas las neolitizaciones en el mundo. Hasta el punto que los primeros arqueólogos al final de los años $50 \mathrm{se}$ interesaron en los procesos americanos, quienes no hicieron más, en un primer momento, que inspirarse en los estudios realizados desde los años 30 en el Próximo Oriente. Procuraban encontrar más paralelismos que evidencias sobre la especificidad de los procesos americanos. Algunos investigadores alcanzaron a proponer que la aparición de la agricultura se debía, sobre todo, a las influencias venidas del Viejo Mundo (Lavallée 2000a). La situación ha cambiado y unas cuantas semejanzas que a veces se observan, no pueden ocultar la originalidad profunda de los procesos americanos, tanto en su ritmo como en el orden de aparición de sus componentes. Además, las investigaciones más recientes demuestran cada día su profundidad temporal, que ahora casi alcanza la del Próximo Oriente. El conjunto de los fenómenos de adaptación selectiva de los grupos humanos a los diversos biotopos ocupados, en relacion al origen de una transformación irreversible de los modos de vida, queda bien comprobado en los Andes desde el 9000 AP, lo que significa que han debido iniciarse mucho más temprano, antes de ser perceptibles en el registro arqueológico.

Esta transición de un mundo de predadores a un mundo de productores y agricultores conforma un tema inagotable de estudio y constituye el origen de una producción bibliográfica enorme. No pretendo aquí aportar algo nuevo, sino tan sólo quisiera transmitir algunas reflexiones a propósito de ciertos aspectos de estos procesos innovadores. En primer lugar, la sedentarización y luego la domesticación animal. En cambio, no me arriesgaré, sino muy brevemente, sobre la domesticación vegetal, por una parte, porque las investigaciones sobre el tema y los problemas planteados - por lo que toca a la identificación de especies domesticadas o no, a los primeros focos de domesticación y también a las vías de difusión de las especies, como de las prácticas hortícolas- superan mis conocimientos. Por otra parte, porque me parece que suscitan más discusiones y polémicas en tanto que todavía no aportan respuestas muy convincentes.

Enfocando el tema de la sedentarización, una de las singularidades de los Andes Centrales es haber dado lugar muy tempranamente a instalaciones, a lo largo del Pacífico, con poblaciones relativamente estables, sin que se haya practicado la agricultura. Es cierto que las primeras ocupaciones humanas detectadas entre el extremo sur de Ecuador y el norte de Chile, que se remontan aproximadamente a 12000 y 10000 AP, no son más que campamentos estacionales de pescadores recolectores marítimos, como La Chimba-13, Acha-2 en Chile, Quebrada Jaguay y Quebrada de los Burros, que estamos estudiando, en el extremo sur de Perú (Lavallée 2000b: 100), o campamentos de cazadores seminómadas establecidos a cierta distancia del océano, como los de Paiján en el norte de Perú (Lavallée 2000b: 90). No son instalaciones permanentes, aunque algunos campamentos parecen haber sido ocupados durante la mayor parte del año. Pero desde los 8000 AP aparecen los primeros asentamientos estables, las 
más tempranas aldeas costeras, como Las Vegas-80 en el sur de Ecuador (Fase Las Vegas, entre 8000 y 6500 AP), y Paloma, en la costa central de Perú (a partir de 7000/6500 AP), ambas situadas en un ambiente natural desértico o semidesértico (ver una síntesis de estos estudios en Lavallée 2000a y b).

La hipótesis que el mar, gracias a la abundancia y estabilidad de sus recursos, habría favorecido un aumento demógrafico y permitido el desarrollo de sociedades costeras relativamente complejas, antes de que fuera practicada la agricultura, fue propuesta por primera vez por Lanning en 1967, desarrollada y argumentada por Fung en 1972, y llevada a su extremo por Moseley en 1975 (Moseley 1975, 1992). Estos planteamientos han suscitado numerosas y, a veces, violentas críticas (Raymond 1981; Wilson 1981; Bonavia 1982, 1998). Sin embargo, en Paloma, que es una instalación aparentemente permanente, conformada por más de un centenar de pequeñas chozas, la mayor parte de la alimentación se obtuvo del mar (Quilter 1989). Si bien es cierto que algunos elementos vegetales, tanto silvestres como cultivados (calabazas y mates, talvez, porotos) están presentes, juegan un rol limitado. Además, el producto de la pesca cuenta con una proporción elevada de pequeñas especies, como sardinas y anchovetas todavía más pequeñas. Nosotros mismos, en Quebrada de los Burros, gracias al trabajo del arqueoictiólogo Philippe Béraez, hemos comprobado en todos los niveles ocupacionales desde 9000 AP (los niveles más antiguos quedan por analizar) la presencia de cantidades notables de vértebras de Clupeidae (sardinas, machetes y anchovetas) en el conjunto de los restos óseos ícticos. Sin embargo, Bonavia (1998: 52) escribía: "Si en teoría no hay problema para aceptar la posibilidad de que se haya explotado la anchoveta en tiempos precerámicos, [...] no hay pruebas arqueológicas que lo sustenten y lo demuestren". En el mismo artículo, sostenía que "el hombre llega a la costa peruana hacia los $8000 \mathrm{AC}$ y vive con tecnología de cazador recolector hasta los 6000 $\mathrm{AC}$ (...). Y si bien pesca, lo hace lanceando y esa, definitivamente, no es una técnica de pescadores" (Bonavia 1998: 56).

Resulta que Bonavia toma como única referencia el Paijanense, complejo cultural de cazadores en el cual, efectivamente, las grandes puntas lanceoladas y muy aguzadas que lo caracterizan parecen haber servido a veces para arponear peces grandes. En cambio, ignora las evidencias de instalaciones costeras de pescadores recolectores de mariscos que demuestran, para la misma época, un dominio perfecto del medio marítimo usando técnicas de pesca sofisticadas, incluyendo el uso de lienzas y anzuelos, redes y muy probablemente embarcaciones (Bonavia 1996a: 181).

Afirma también Bonavia (1996a: 59) que: "los hombres que bajaron a la costa ya traían consigo los conocimientos relativos a las plantas". ¿Y si los hombres no hubieran "bajado" desde las tierras altas hacia la costa y que se hubiera tratado de otros grupos humanos venidos siguiendo otras vías? ¿Y si grupos perfectamente adaptados al medio marítimo desde un largo tiempo hubieran sido capaces de desarrollar un modo de vida estable y perenne, sin recurrir a otros recursos vegetales que los silvestres y de manera complementaria? No pretendo aquí responder a estas preguntas y concluir el debate. Pero me parece que, a la luz de los descubrimientos recientes en sitios costeros, se abre un amplio campo de investigación y de reflexión y que una palabra que nosotros, arqueólogos, deberíamos siempre evitar de emplear es aquella "definitivamente". De todas formas, no se puede negar que una verdadera agricultura sólo se practicará en la costa alrededor de 6000/5500 AP y no se volverá preponderante sino mucho más tarde.

Otras regiones en el mundo han conocido evoluciones comparables, sino en sus desarrollos, en los mismos términos generales. En el Próximo Oriente, por ejemplo, existe una sedentarización también mucho antes de la agricultura y del pastoreo. Las primeras aldeas del Natufiense aparecen entre los 12000 y 10500 AP, cuando la vegetación esteparia deja lugar a una sabana rica en cereales silvestres que cosechan cazadores recolectores que ya se han vuelto sedentarios. Como en Perú, la primera innovación la constituye la aldea. Y, en ambos casos, los hombres aprovecharon un medio natural capaz de ofrecer un sustento seguro: fue el mar en el Area Central Andina, la sabana en el Creciente Fértil.

Durante el mismo tiempo, parece que varias experiencias hortícolas se llevaron a cabo muy temprano, talvez desde el inicio del Holoceno, sobre la vertiente oriental de la cordillera y, poco más tarde, en los valles y cuencas interandinas de altura media. Fechas muy antiguas -entre 10600 y $7500 \mathrm{AP}-$ se obtuvieron en los años 80 , para restos de plantas que los botánicos estimaron cultivadas, provenientes de la cueva de Guitarrero, en el Callejon de Huaylas (Perú) (Lynch 1980; Lynch et al. 1985). 
Sin embargo, estas fechas fueron recientemente puestas en duda y se procedió a su reevaluación, gracias a la utilización de análisis radiométricos por espectrometría de masa con acelerador (AMS), que requiere de cantidades mínimas, permitiendo que se fecharan los mismos restos vegetales y no muestras de carbón procedentes de los niveles arqueológicos supuestamente asociados. Hoy en día los restos de ají (Capsicum chinense) de Guitarrero quedan como los más antiguos testimonios de vegetales cultivados en los Andes Centrales, pero sabemos que los restos de pallares (Phaseolus lunatus) y de porotos (Phaseolus vulgaris) son muchos más recientes, con fechas que no superan 5000-4400 AP (Kaplan 1995 Ms).

De todas maneras, sabemos que cuando una planta, presente en el registro arqueológico, está identificada por los botánicos como "domesticada", testimonia una fase avanzada de un proceso iniciado mucho antes. En los Andes Centrales este proceso parece haber empezado a fines del Pleistoceno. De tal manera que serían las sociedades de cazadores recolectores quienes habrían iniciado esta domesticación, practicando de manera consciente o no la selección de ciertas especies. Esto para subrayar una vez más, si fuera necesario, hasta qué punto la neolitización fue el resultado de un largo y lento continuum.

En contraste con la diversidad de las plantas cultivadas, sabemos que sólo cuatro especies animales fueron domesticadas en el Area Andina: un pato (Cairina moschata), el cuy (Cavia porcellus) y dos especies de camélidos silvestres, hoy en día desaparecidos, pero ancestros de los actuales guanaco y vicuña. No obstante, sólo los camélidos jugarán desde el inicio un papel económico mayor. Hasta ahora el único yacimiento andino donde se ha podido documentar con datos arqueozoológicos concretos la transición de la caza a la crianza de los camélidos sigue siendo, que yo sepa, el abrigo de Telarmachay en la Puna de Junín (Perú). En efecto, a base de los patrones de utilización de los animales a lo largo de varios milenios, se ha podido evidenciar un desarrollo in situ, desde una caza generalizada de los ungulados (cérvidos y camélidos salvajes) entre 9500 y 7000 AP, hacia la especialización de la caza de sólo camélidos entre 7000 y 6000 AP hasta, por fin, la aparición de los primeros animales domesticados entre $6000 \mathrm{y}$ 5500 AP. Sin entrar en los detalles de los análisis realizados por J. Wheeler, en el marco de nuestro proyecto de investigaciones en la Puna de Junín, recordaré que la determinación de la presencia de animales domésticos se basó en el incremento de la frecuencia de restos de camélidos, en los cambios en las curvas de supervivencia y en la morfología dental. Desde la publicación de la monografia de Telarmachay (Lavallée et al. 1985, 1995), esta hipótesis ha sido criticada (Kent 1982) y se le han opuesto las ideas de Rick (1980), quien ha excavado el abrigo vecino de Pachamachay, proponiendo una fecha mucho más tardía para la aparición de camélidos domésticos. Sin embargo, como lo recuerda Bonavia (1996b: 243), hoy en día hay un amplio consenso para pensar que la domesticación inicial comenzó efectivamente hace unos 6000 años AP, y hay también un acuerdo en admitir que la alpaca ha sido el primer camélido doméstico. A pesar de la discrepancia que existe entre las dos interpretaciones, Telarmachay y Pachamachay quedan hasta la fecha como los dos únicos sitios de los Andes Centrales que han proporcionado datos precisos, y parece que sería necesario analizar otros sitios de gran altura entre Junín y la cuenca del lago Titicaca. Es de lamentar también que nunca se hayan publicado los informes faunísticos del Proyecto Ayacucho dirigido por R. Macneish en los años 70, y que tampoco se haya llevado a su término el análisis completo del material faunístico de los niveles más antiguos de Telarmachay.

De acuerdo a Yacobaccio (2000: 278), la domesticación comenzó en un contexto de cazadores complejos "que tenían control de segmentos poblacionales de camélidos en territorios relativamente fijos". Ahora bien, creo que es precisamente lo que hemos constatado en Telarmachay donde, en un contexto de caza especializada e intensiva al interior de un territorio relativamente reducido, tenemos indicios que cierta cantidad de animales eran guardados en corrales. Por otra parte, existe una diferencia de opinión sobre el (o las) área(s) geográfica(s) en que se produjo de manera independiente la domesticación de los camélidos. Aparte de la Puna Central andina donde parece firmemente comprobada, varias otras áreas han sido propuestas. Por un lado, la cuenca del Titicaca, aunque siguen faltando evidencias arqueológicas. Por otro, la región de Atacama en el norte de Chile, donde se ha identificado el campamento Puripica-1 (entre 4800 y 4100 AP) con camélidos grandes que, según Hesse (1986), "serían" componentes de un proceso de domesticacion local. Observando esta vez el perfil de edad de la muestra de fauna procedente de este sitio y sus contextos culturales, Núñez (1992) piensa que sus ocupantes fueron cazadores y pastores iniciales; sin embargo, 
Yacobaccio (2000: 270-271) considera más prudente explicar la evidencia como manejo de protección de segmentos poblacionales de camélidos. Posiblemente también la Puna del Noroeste Argentino, aunque aquí también faltan indicios concretos. En efecto, en el sitio Quebrada Seca-3, sólo un analisis de fibras de lana ha permitido "identificar fibras análogas a una variedad de llama actual (...) presentes en el registro arqueológico desde momentos muy tempranos (o sea, anteriores al 8000 AP)" (Olivera y Elkin 1994: 107). ¿Serán realmente suficientes estas observaciones para comprobar la existencia de focos independientes de domesticación? Esta hipotesis, por cierto, es lógica y probable, pero la hipótesis alternativa y más "clásica" de una transferencia de las prácticas pastoriles desde los Andes Centrales todavía no ha sido invalidada formalmente.

Queda, por fin, una pregunta fundamental: ¿Por qué, mientras existía un sistema de subsistencia basado en un aprovechamiento racional de la fauna salvaje, los hombres lo han abandonado poco a poco por los apremios inherentes a la domesticación? Propusimos en 1990 una explicación, escribiendo:

“(...) la hipótesis de una presión del medio natural parece poder ser eliminada (...) podemos imaginar que esta domesticación se produjo casi al azar y de manera no premeditada, siendo la preocupación principal de los cazadores explotar lo mejor posible los rebaños salvajes. Una caza cada vez más intensiva y cada vez más selectiva de estos animales y el profundo conocimiento de su comportamiento (muy gregario y ocupando un territorio estable) que se deriva, habría generado una suerte de comensalismo, donde camélidos y hombres vivían casi en simbiosis, sin que la libertad de los primeros y la disponibilidad de los segundos se vean entorpecidas. Los efectos benéficos de esta asociación y de la acción del hombre (selección de los animales a beneficiar, eliminación de los individuos agresivos o enfermos, protección de las hembras y vigilancia de la parición, entre otros) fueron probablemente percibidos con rapidez, y los gestos para recrearlos memorizados y repetidos de generación en generación" (Lavallée 1990: 40-41).

Propusimos entonces, hablar de una "innovación conservadora", vista la forma en la cual el modo de vida que implicó la domesticación incipiente se integraba hasta confundirse con los comportamientos ancestrales de los cazadores. La caza, además, continuó jugando un rol importante, en la medida en que, al menos en un inicio, las bajas operadas en el rebaño doméstico debían ser subordinadas al cuidado de aumentarlo.

Independiente de cuáles hubieran sido el incentivo y el primer foco, la domesticación de los camélidos representa una etapa fundamental en la trayectoria cultural andina, pues las dos especies así "creadas" por el hombre van a jugar, al igual que el maíz para el desarrollo de las sociedades costeras, un papel primordial en el desarrollo de las sociedades cordilleranas. Sin embargo, debería pasar mucho tiempo antes que, en las tierras altas, una economía agropastoril logre soportar un aumento demográfico y una organización social equivalente a los logros costeños conocidos desde varios milenios gracias a la agricultura.

Para terminar, no haré más que tocar someramente un problema aún si resolver (¡uno más!), el de la "invención" de la cerámica en América. En realidad, creo que no deberíamos incluir esta invención en los procesos de neolitización. Ahora sabemos muy bien que, en varios puntos del mundo, la aparición de una economía de producción antecedió en varios milenios a la fabricación y uso de recipientes de barro cocido. En el Próximo Oriente, donde se manipulan los cereales desde $10000 \mathrm{AP}$, la cerámica más antigua no aparece sino hacia 7000 AP. En Africa (entre Egipto y Níger), se cuenta ahora con evidencias de producción cerámica en un contexto de grupos viviendo de caza, pesca y recolección de plantas silvestres, desde aproximadamente 9000 AP. Y ¿qué decir del Japón, donde la cerámica se conoce desde los 12000 AP, utilizada por sociedades no productoras de alimentos y que lo seguirán siendo durante miles de años más?

Volviendo a nuestra América, que si la cerámica fue inventada en varios lugares o difundida desde un centro único, es una cuestion que todavía no tiene una respuesta definitiva. La cerámica más antigua por ahora conocida proviene del corazón mismo de la Amazonia (en Taperinha, ca. 7000 AP), superando por más de mil años los vestigios bien conocidos encontrados en el litoral de Colombia (Monsú, Puerto Hormiga, Puerto Chacho), y aun los más recientemente descubiertos por Oyuela-Caycedo en la localidad de San Jacinto-I y fechados entre 6000 y 5300 AP (Oyuela-Caycedo 1993, 1995). Lo interesante es que, al igual de los ejemplos que mencioné para el Viejo Mundo, en San Jacinto la cerámica se utilizaba en un contexto de grupos 
cazadores recolectores móviles que explotaban recursos vegetales estacionales (Oyuela-Caycedo 1995). Debemos entonces interrogarnos sobre la función de estas cerámicas preagrícolas, que no era de manera obligatoria una función culinaria o de almacenamiento. Varios autores han propuesto la hipótesis de una función social, constituyendo los recipientes de alfarería bienes de prestigio y/o de intercambio. Según Pratt (1999), quien ha analizado el conjunto cerámico de San Jacinto, estos tiestos pudieron haber sido utilizados mayormente con el motivo de acontecimientos festivos, banquetes, y muy poco para la cocción o la preparación de alimentos. Esto, por supuesto, es algo especulativo, pero talvez valdría la pena realizar análisis estadísticos semejantes sobre otros conjuntos cerámicos antiguos de los Andes Centrales como, por ejemplo, el de Tutishcainyo en el valle del Ucayali. Otro punto notable es que ninguna de estas cerámicas antiguas refleja una tecnología lo suficientemente primitiva para ser interpretada como el resultado de una invención local. Su diversidad más bien reflejaría procesos independientes. De este punto de vista, los hallazgos de A. Roosevelt en Taperinha (1999) militan en favor de un origen amazónico, lo que al final confirmaría las intuiciones de Lathrap postuladas desde los años 70. En los Andes Centrales la cerámica es más bien tardía en comparación con los ejemplos mencionados; aparece casi siempre en contexto aldeano, alrededor de los 4000 AP y, muy probablemente, fue introducida desde otras regiones, desde los Andes Septentrionales o las regiones amazónicas. Lo importante, a nuestro parecer, es que el uso de recipientes de barro cocido, si bien representó un avance técnico importante, no implicó un trastorno fundamental del modo de vida de aquéllos que los inventaron o los adoptaron, sino que, más bien, facilitó el transporte, la preparación culinaria y el almacenamiento de alimentos y de otros productos. Pero varios procedimientos de almacenamiento fueron empleados con anterioridad, como la utilización de pozos o silos cavados y la de cesterías, incluyendo recipientes de calabaza, de cuero o de piedra. Nuevamente, lo mismo ha sido observado en el Próximo Oriente, donde el uso de recipientes de piedra antecede a la mutación definitiva de las estrategias alimenticias. Sobre este tema también parece que queda mucho por investigar.

Para concluir, y por ser francesa, quisiera subrayar una diferencia fundamental que separa, o mejor dicho, separaba hasta hace poco los estudios sobre la neolitización en Europa y en América. En Europa, la investigación sobre lo llamado "Neolítico" ha sido durante un largo tiempo considerado como "el pariente pobre". Se le indicaba como la etapa final de un preeminente Paleolítico, como una suerte de epifenómeno casi desprovisto de interés. Tal visión resultaba de un magistral error conceptual, cuando se trata al revés de la elaboración de un mundo nuevo, de una relación con el medio ambiente totalmente renovado y de la construcción de un nuevo sistema de valores. Tener la suerte, como la he tenido, de trabajar en una "área nuclear" de un potencial tan rico como el de los Andes me ha permitido, al menos lo espero, tomar la exacta dimensión de lo que, lejos de ser un final, fue el inicio de unas de las trayectorias culturales más originales y fecundas del mundo.

\section{REFERENCIAS CITADAS}

BONAVIA, D., 1982. Los gavilanes. Mar, desierto y oasis en la historia del hombre. COFIDE, Instituto Arqueológico Alemán, Lima.

—1996a. De la caza-recolección a la agricultura: Una perspectiva local. Boletín del Instituto Francés de Estudios Andinos 25 (2): 169-186.

_ 1996b. Los camélidos sudamericanos (una introducción a su estudio). Trabajos del Instituto Francés de Estudios Andinos, Tomo 93, Lima.

_ 1998. ¿Bases marítimas o desarrollo agrícola? En 50 Años de estudios americanistas en la Universidad de Bonn, BAS, 30: 45-62.
BRAIDWOOD, R., 1960. The agricultural revolution. Scientific American 203: 130-148.

FUNG, R., 1972. El temprano surgimiento en el Perú de los sistemas sociopolíticos complejos: Planteamiento de una hipótesis de desarrollo original. Apuntes Arqueológicos 2: $10-32$.

HERITIER, F., 2001. Innovation, invention, découverte. $L a$ Géographie-Acta Geografica 1503: 5-22.

HESSE, B., 1986. Buffer resources and animal domestication in prehistoric Northern Chile. En Archaeozoologia, Mélanges publiés à l'occasion du $5^{e}$ Congrès International d'Archéozoologie, pp. 73-86. Editorial La Pensée Sauvage, París. 
KAPLAN, L., 1995 Ms. Phaseolus beans, accelerator dates in the Americas. Ponencia presentada al $60^{\circ}$ Congreso Anual de la Society for American Archaeology, Minneapolis.

KENT, J., 1982. The domestication and exploitation of the south american camelids: Methods of analysis and their application to circum-lacustrine archaeological sites in Bolivia and Peru. $\mathrm{Ph}$. D. Dissertation, Washington University, St. Louis.

LANNING, E. P., 1967. Peru before the incas. Prentice Hall, Englewood Cliffs.

LAVALLEE, D., 1990. La domestication animale en Amérique du Sud. Le point des connaissances. Boletín del Instituto Francés de Estudios Andinos 19 (1): 25-44.

2000a. The first south americans. The University of Utah Press, Salt Lake City.

_2000b Les premiers producteurs de l'Amérique du Sud. En Premiers paysans du monde, J. Guilaine (Ed.), pp. 191-211. Editorial Errance, París.

LAVALLEE, D., M. JULIEN, J. WHEELER y C. KARLIN, 1985. Telarmachay. Chasseurs et pasteurs préhistoriques des Andes. Travaux de l'Institut Français d'Études Andines. Recherche sur les civilizations "synthèse" 20, París.

_ 1995. Telarmachay. Cazadores y pastores prehistóricos de los Andes. Travaux de l'Institut Français d'Études Andines, Tomo 88, Lima.

LYNCH, T. (Ed.), 1980. Guitarrero cave. Early man in the Andes. Academic Press, Nueva York.

LYNCH, T., R. GILLESPIE, J. GOWLETT y R. HEDGES, 1985. Chronology of Guitarrero Cave, Peru. Science 229: 864-867.

MOSELEY, M., 1975. The maritime foundations of andean civilizations. Cummings Publishing Company, Menlo Park.

-1992. Maritime foundations and multilinear evolution: Retrospect and prospect. Andean Past 3: 5-42.

NUÑEZ, L., 1992. Ocupación arcaica en la Puna de Atacama: Secuencia, movilidad y cambio. En Prehistoria sudameri- cana. Nuevas perspectivas, B. Meggers (Ed.), pp. 283-307. Taraxacum, Washington D. C.

OLIVERA, D. y D. ELKIN, 1994. De cazadores y pastores: El proceso de domesticación de camélidos en la Puna Meridional andina. Zooarqueología de Camélidos 1: 95-124.

OYUELA-CAYCEDO, A., 1993. Sedentism, food production, and pottery origins in the Tropics: The case of San JacintoI. Ph. D. Dissertation, University of Pittsburgh, University Microfilms, Ann Arbor.

1995. Rock versus clay: The evolution of pottery technology in the case of San Jacinto-I, Colombia. En The emergence of pottery: Technology and innovation in ancient societies, W. Barnette y J. Hoopes (Eds.), pp. 133-144. Smithsonian Institution Press, Washington D. C.

PRATT, J. A., 1999. Determining the function of one of the new world's earliest pottery assemblages: The case of San Jacinto, Colombia. Latin American Antiquity 10 (1): 71-85.

QUILTER, J., 1989. Life and death at Paloma: Society and mortuary practices in a Preceramic Peruvian village. University of Iowa Press, Iowa City.

RAYMOND, J. S., 1981. The maritime foundations of andean civilization: A reconsideration of the evidence. American Antiquity 46 (4): 806-821.

RICK, J., 1980. Prehistoric hunters of the high Andes. Academic Press, Nueva York.

ROOSEVELT, A., 1999. The maritime, highland, forest dynamic and the origins of complex culture. En The Cambridge History of the native peoples of the Americas, F. Salomon y S. Schwartz (Eds.), T 1, pp. 249-264. Cambridge University Press, Nueva York.

WILSON, D. J., 1981. Of maize and men: A critique of the maritime hypothesis of State origins in the coast of Peru. American Anthropologist 83 (1): 93-120.

YACOBACCIO, H., 2000. Cazadores complejos y domesticación de camélidos. En El uso de los camélidos a través del tiempo, G. Mengoni, D. Olivera y H. Yacobaccio (Eds.), pp. 261-282. Editorial del Tridente, Buenos Aires. 